\title{
Pediatric epidural extra-osseous swing's sarcoma - extremely rare case report and review of the literature
}

\begin{abstract}
Primary extra osseous Ewing sarcoma is a rare occurrence, even uncommon is presenting as a primary focus in the spinal canal. ${ }^{1}$ They often present with specific focal neurological signs and symptoms of myelopathy or radiculopathy. There are no classic characteristic imaging findings and mimics other common tumors and thus the radiologist must keep an index of suspicion. Diagnosis can only be definitively made by histopathology. Here, we report a 12-year-old girl who complained of bilateral lower limb weakness for nearly a week. MRI showed one solitary well-defined oblong posterior epidural soft tissue lesion at the level of C7-D5. She underwent laminectomy of D2 and complete resection of the tumor. The histopathology confirmed a diagnosis of Ewing sarcoma. Her symptoms and neurological deficits recovered well and the child is undergoing adjuvant chemotherapy and radiotherapy.
\end{abstract}

Volume 4 Issue $3-2017$

\author{
Gopinath, Seena CR, Prashant Moorthy, \\ Kulasekaran N \\ Saveetha Medical College, India
}

Correspondence: Prashant Moorthy, Saveetha Medical College, Saveetha Nagar,Thandalam Kancheepuram-602105,Tel 98844I5538,Email drprashantmdrd@gmail.com

Received: August 18, 2017 | Published: October 02, 2017

Keywords: diagnosis, histopathology, laminectomy, ewing sarcoma

\section{Introduction}

Ewing sarcoma is a bone tumor that has a predilection for long bones in teenagers. It accounts to approximately $6-10 \%$ of all primary bone tumors in children; however, it seldom occurs outside of the bone, and very rarely in the epidural space. ${ }^{1}$ Ewing sarcoma is composed of uncharacterized mesenchymal cells. The most common sites are the chest wall, paravertebral muscles, extremities, and retroperitoneal space; extra skeletal Ewing's sarcoma (extra- osseous form) was first described by Tefft et al., ${ }^{1}$ and the involvement of the epidural space remains rare. ${ }^{2}$ Only very few cases have been reported in literature (Table 1). An additional case of epidural Ewing's sarcoma is reported, and the literature is being reviewed.

Table I Only very few cases have been reported in literature

\begin{tabular}{llllll}
\hline Case (Ref.) & Age (Years)/gender & Level & Resection & Adjuvant treatment & Outcome(Months) \\
\hline 1 & 6/F & L4 & Partial & CT, RT & DOD (48) \\
$2(3)$ & I7/M & SI-2 & N/A & None & DOD (I) \\
$3(3)$ & 20/M & T2-5 & Partial & CT, RT & DOD (I3) \\
$4(3)$ & I8/F & L5 & N/A & CT, RT & DOD (7) \\
$5(5)$ & I8/M & LI & Complete & CT, RT & NED (I6) \\
$6(5)$ & 27/F & T6 & Complete & CT, RT & NED (I20) \\
$7(6)$ & 23/M & L5-SI & Partial & CT, RT & DOD (I2) \\
$8(7)$ & L2-3 & Partial & CT, RT & NED (6) \\
$9(8)$ & L3/M & N/A & CT, RT & NED (I5) \\
$10(12)$ & I2/M & L4-S2 & Partial & CT, RT & DOD (I6) \\
II (I3) & IO/M & L3 & Partial & CT, RT & DOD (8) \\
$12(14)$ & I7/M & TII & Partial & CT, RT & DOD (42) \\
$13(15)$ & I8/M & LI & Complete & None & DOD (5) \\
$14(16)$ & 4/M & TI2-LI & Partial & CT & NED (40) \\
$15(17)$ & T/M & TI2-L3 & N/A & N/A & N/A \\
$16(18)$ & I5/F & C7-TI & Partial & CT & NED (48) \\
\hline
\end{tabular}


Table Continued.

\begin{tabular}{|c|c|c|c|c|c|}
\hline Case (Ref.) & Age (Years)/gender & Level & Resection & Adjuvant treatment & Outcome(Months) \\
\hline $17(18)$ & $38 / M$ & C5-7 & Partial & $\mathrm{CT}$ & AWD (5) \\
\hline $18(19)$ & $24 / M$ & $\mathrm{Cl}-5$ & Partial & $\mathrm{CT}, \mathrm{RT}$ & NED (25) \\
\hline $19(20)$ & $29 / F$ & C3-5 & Partial & $\mathrm{CT}, \mathrm{RT}$ & NED (30) \\
\hline $20(20)$ & I8/M & T8 & Partial & $\mathrm{CT}, \mathrm{RT}$ & AWD (18) \\
\hline $21(20)$ & $22 / M$ & L5-SI & Partial & $\mathrm{CT}, \mathrm{RT}$ & NED (I5) \\
\hline $22(20)$ & $31 / M$ & L3-4 & Partial & $\mathrm{CT}, \mathrm{RT}$ & NED (32) \\
\hline $23(20)$ & $13 / M$ & C3-5 & Partial & $\mathrm{CT}, \mathrm{RT}$ & NED (II) \\
\hline $24(2 \mathrm{I})$ & $33 / M$ & $\mathrm{~T} 4-7$ & Complete & $\mathrm{CT}, \mathrm{RT}$ & NED (3) \\
\hline $25(22)$ & $12 / F$ & TI-3 & Partial & $\mathrm{CT}, \mathrm{RT}$ & DOD (32) \\
\hline $26(23)$ & $7 / F$ & $C 2-4$ & Complete & $\mathrm{CT}, \mathrm{RT}$ & NED (60) \\
\hline $27(24)$ & $\mid 5 / F$ & C3-7 & Partial & N/A & N/A \\
\hline $28(25)$ & $13 / M$ & T9 & Partial & $\mathrm{CT}, \mathrm{RT}$ & AWD (9) \\
\hline $29(26)$ & I8/M & C6-TI & Complete & $\mathrm{CT}$ & NED (I3) \\
\hline $30(1)$ & $37 / F$ & T8-9 & Partial & $\mathrm{CT}, \mathrm{RT}$ & DOD (22) \\
\hline $3 I(27)$ & $53 / M$ & $\mathrm{C} 2-\mathrm{C} 7$ & Complete & $\mathrm{CT}, \mathrm{RT}$ & NED \\
\hline 32(THIS CASE) & $12 / F$ & C7-D5 & Complete & $\mathrm{CT}, \mathrm{RT}$ & TREATMENT \\
\hline
\end{tabular}

M, male; F, female; C, cervical;T, thoracic; L, lumbar; CT, chemotherapy; RT, radiotherapy; PBSCT, peripheral blood stem cell transplantation; DOD, dead of disease; NED, no evidence of disease;AWD, alive with disease; N/A, not available

\section{Case report}

A 12year-old child presented with progressive weakness of bilateral lower limbs for 2 weeks. No history of fever or trauma was present. Bowel and bladder habits were normal. Clinical examination revealed decrease in power on bilateral lower limbs (power of 2/5). Laboratory findings were within normal limits. Magnetic resonance imaging (MRI) of lumbar spine was normal and a whole spine screening was planned which showed a well defined vertically oriented extra medullary lesion appearing homogeneously hypo intense on $\mathrm{T} 1$ and iso to hyperintense on T2 extending across C7 to D5 vertebral levels causing compressive displacement of dorsal cord anteriorly with resultant mild cord edema. On DWI, the lesion, spinous process and bilateral pediculo-laminar complex of D2 vertebral body show significant areas of restricted diffusion -suggestive of lesion extension into the spinoligamentous complex and pediculo-laminar complex of D2 vertebral body. Mild bone irregularity was seen in D2 spinous process.

Laminectomy at D2 and complete resection of the tumor were performed. Histopathology showed small round cells with hyperchromatic nuclei and scant cytoplasm. Fragments of tissue showed a tumor composed of sheets of cells with scant cytoplasm and uniform round hyperchromatic nuclei with tumor cells infiltrating into surrounding adipose tissue leading to diagnosis of epidural ewing's sarcoma. After surgery the child was planned for chemo radiotherapy. She recovered remarkably well. First, she regained movement in his lower extremities and subsequently regained antigravity ability. Bilateral lower limb power was $4 / 5$. The child is undergoing chemotherapy cycles currently.

\section{Discussion}

Ewing's sarcomas arising from soft tissues are termed as extra skeletal ewing's sarcoma (EES). Most EES patients are between 10 and 30years of age, with a peak incidence at around 20years of age. ${ }^{3,4}$ Hamby (1935) reviewed an exhaustive literature of intraspinal tumours of childhood, consisting of 214 cases, of which $41 \%$ involved the epidural space. The relative frequency of tumour types in this location was as follows: sarcomas $(44 \%)$, others were schwannomas, dermoid cysts (6), sympathetic tumours (5), vascular (4), fibrous tumours (3), meningiomas (2), and lipomas (2). ${ }^{5}$

The most common sites are extremities, paravertebral region and retroperitoneal space. ${ }^{4-16}$ However, a number of cases have been reported to arise in numerous locations such as the skin, larynx, esophagus, and paravertebral region. ${ }^{4}$ Only very few cases (Table 1) of EES arising in the spinal epidural space, including our case, have been reported in the literature. The mean presentation age is around 19 years. The male-female relationship is 1.5:1 in spinal epidural EES resembles that of bone (Figure 1-5). ${ }^{17-26}$

The most common presentation is back pain, radicular pain, and paresis of unilateral or bilateral limbs, sensory disturbances, and bladder dysfunction. All these symptoms are related to compression of the cord, nerve roots and cauda equine as seen with other epidural tumors. The most helpful imaging studies for diagnosis are MR and CT. CT may reveal hypo dense mass displacing the thecal sac on myelography, calcifications if any and bone changes. On post contrast study the lesions show homogenous enhancement. MR is the modality of choice and usually presents as T1 iso-hypo and T2 hyperintense lesion with or without extension into the neural foramen. On post contrast study the lesions show homogenous enhancement and sometimes may show a dural tail. Differentials include nerve sheath tumors, meningioma, hematoma, hemangioma, lymphoma, and leukemia.

Blood investigations will exclude leukemia. Bone changes \& 
GRE sequences excluded hematoma. Schwannoma, meningioma, were considered due to the imaging features favoring these two and metastases was considered a distinct possibility. Epidural EES is particularly very unusual only to present with soft tissue component, and it was not initially considered in the differential diagnosis before histopathology.
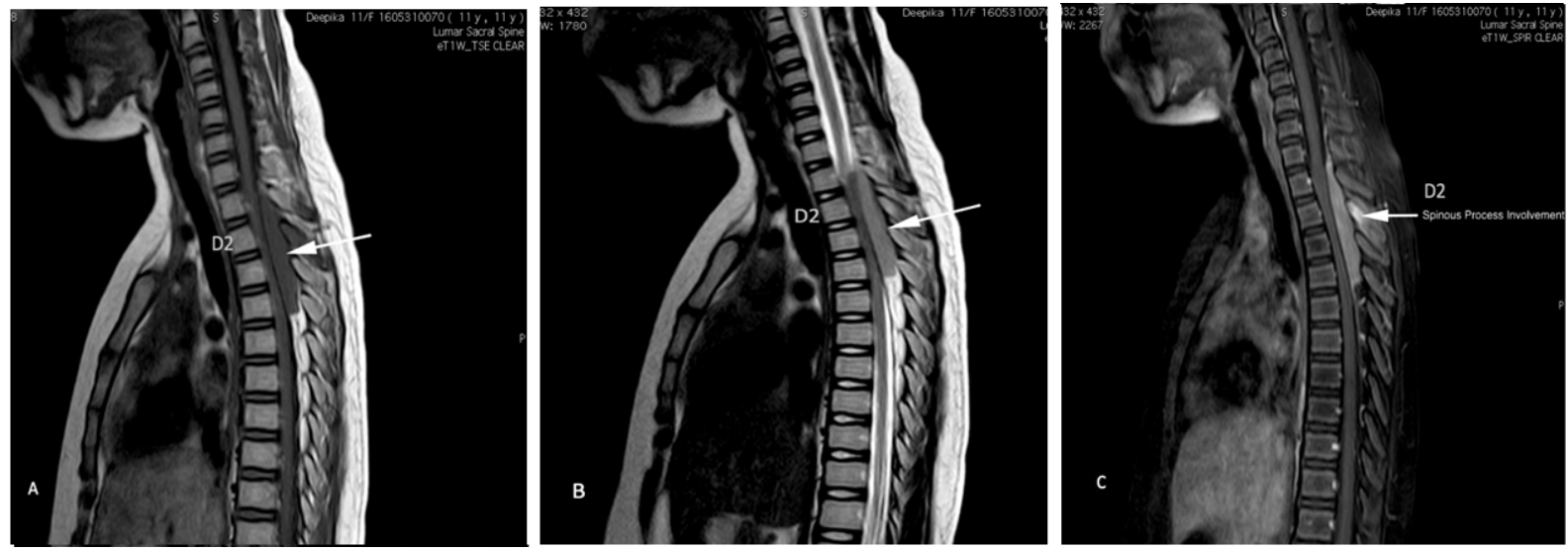

Figure I MRI findings:

A. TI-weighted sagittal imaging.

B. T2-weighted axial imaging shows a circumscribed, posterior epidural tumor at the C7 to D5 level that is TI hypointense and T2 isointense and is causing compressive cord edema and foraminal widening at D2-D3 and D3-D4 levels.

C. On post contrast study the lesion shows significant homogenous enhancement with varying degrees of foraminal extension at D2-D3 and D3-D4 level. Enhancing thin dural tail noted along cranial and caudal aspects of the lesion. Enhancing marrow edema noted in the spinous process of D2 vertebra - suggestive of lesion extension. Enhancement noted in the spinoligamentous complex and pediculolaminar complex of D2 vertebral body.

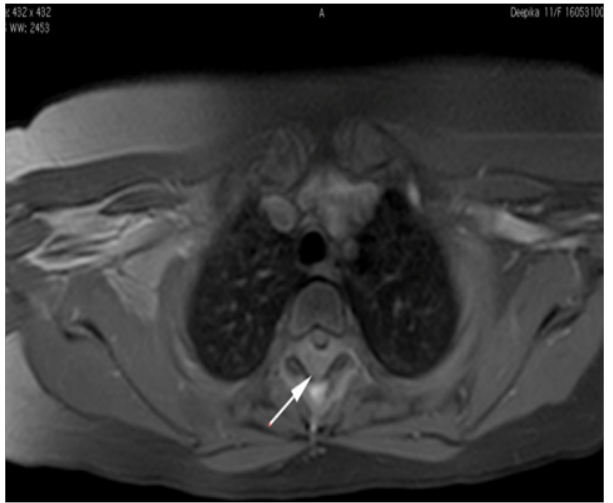

Figure 2 Post contrast axial section showing tumor displacing the cord anterolaterally.

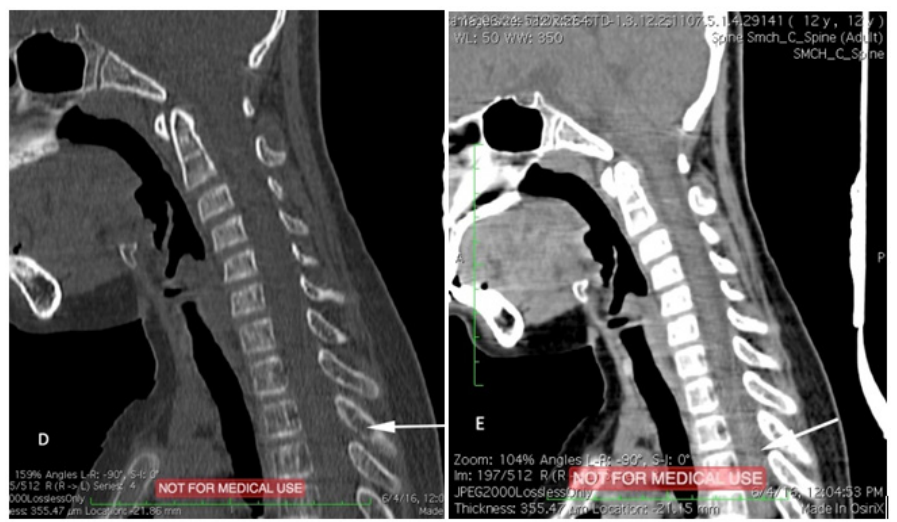

Figure 3 CT images shows: Lesion is iso to hypodense without any intra lesional calcifications. Cortical breech noted along the inferior aspect of the D2 spinous process.
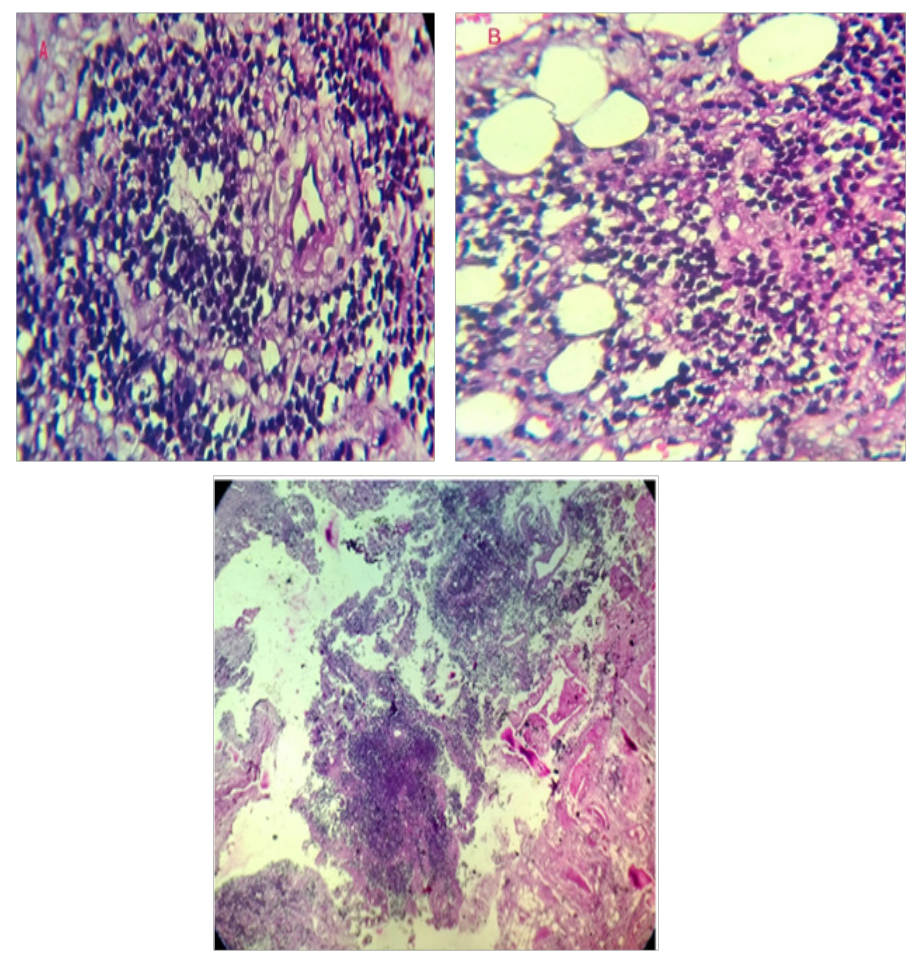

Figure 4A \& B High power view showing small round cells with hyperchromatic nuclei and scant cytoplasm. Tumor cells are seen to infiltrate the surrounding adipose tissue.

Figure 4C Scanner view showing sheets of tumor cells which are small and uniformly round.

Histopathlogy description: Section shows fragments of tissue showing a neoplasm composed of sheets of cells with scant cytoplasm and uniform round hyperchromatic nuclei with tumor cells infiltrate into surrounding adipose tissue -represents Extraskeletal ewings sarcoma.

Citation: Gopinath, Seena CR, Moorthy P, et al. Pediatric epidural extra-osseous swing's sarcoma - extremely rare case report and review of the literature. Int J Radiol Radiat Ther. 2017;4(3):370-373. DOI: 10.15406/ijrrt.2017.04.00096 
The hisopathology of small round cell malignant tumors is based upon the immunohistolgy and ultra structural analysis. ${ }^{9}$ Histopathological EES is classified as a small round cell tumor because of the uniform small round cell appearance. Such small round cell tumors may be sometimes misdiagnosed as lymphoma, metastases synovial sarcoma. Therefore, immuno- histochemical analysis is necessary for further tumor classification. Approximately 95\% of EESs express membranous immuno- reactivity for CD99 (MIC2). After the diagnosis a laminectomy and resection should be done followed by chemotherapy and radiotherapy for long-term disease free survival. ${ }^{10,11}$

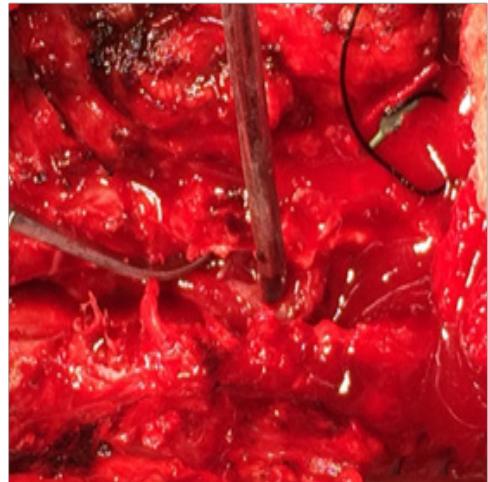

Figure 5 Intraoperative pictures showing resection of the epidural lesion.

\section{Conclusion}

We have reported a very rare case of epidural EES. Imaging features are difficult to distinguish it from other common nerve sheath tumors, metastatic tumor or lymphoma. Although rare, possibility of EES should be kept in mind in providing a differential diagnosis especially in younger age group. Biopsy or resection is always needed in all cases to confirm the diagnosis and allowing appropriate management

\section{Acknowledgements}

None.

\section{Conflict of interest}

Author declares that there is no conflict of interest.

\section{References}

1. Tefft M, vawter GF, Mitus A. Paravertebral 'round cell' tumors in children. Radiology. 1969;92(7):1501-1509.

2. Yasuda T, Suzuki K, Kanamori M, et al. Extraskeletal ewing's sarcoma of the thoracic epidural space: case report and review of the literature. Oncology Rep. 2011;26(3):711-715.

3. Angervall L, Enzinger FM. Extraskeletal neoplasm resembling Ewing's sarcoma. Cancer. 1975;36(1):240-251.

4. Weiss SW, Goldblum JR. Extraskeletal Ewing's sarcoma/primitive neuroectodermal tumor family. In: Weiss SW, Goldblum JR editors. Enzinger and Weiss's Soft Tissue Tumors. 5th ed. USA: Mosby; 2007. p. 963-979.

5. Scheithauer BW, Egbert BM. Ewing's sarcoma of the spinal epidural space: report of two cases. J Neurol Neurosurg Psychiatry. 1978;41(11):1031-1035.

6. Mahoney JP, Ballinger WE, Alexander RW. So-called extraskeletal Ewing's sarcoma. Report of a case with ultra- structural analysis. Am J ClinPathol. 1978;70(6):926-931.
7. Fink LH, Meriwether MW. Primary epidural Ewing's sarcoma presenting as a lumbar disc protrusion. J Neurosurg. 1979;51(1):120-123.

8. Simonati A, Vio M, Iannucci AM, et al. Lumbar epidural Ewing sarcoma. Light and electron microscopic investigation. J Neurol. 1981;225(1):67-72.

9. Kennedy JG, Eustace S, Caulfield R, et al. Extraskeletal Ewing's sarcoma: a case report and review of literature. Spine. 2000;25(15):1996-1999.

10. Folpe AL, Goldblum JR, Rubin BP, et al. Morphologic and immunophenotypic diversity in Ewing family tumors: a study of 66 genetically confirmed cases. Am J Surg Pathol. 2005;29(8):1025-1033.

11. Lim TC, Walter Tang LT, Yoke Sun Lee. Congenital extraskeletalewing's sarcoma of face: a case report. Journal of the Science and Specialties of the Head and Neck. 1994;16(1):75-78.

12. Spaziante R, de Divitiis E, Giamundo A, et al. Ewing's sarcoma arising primarily in the spinal epidural space: fifth case report. Neurosurgery. 1983;12(3):337-341.

13. Ruelle A, Boccardo M. Primary extra-skeletal Ewing's sarcoma mimicking a disc protrusion. Neurochirurgia (Stuttg). 1987;30(4):125-126.

14. Sharma BS, Khosla VK, Banerjee AK. Primary spinal epidural Ewing's sarcoma. Clin Neurol Neurosurg. 1986;88(4):299-302.

15. Machin Valtueña M, Garcia-Sagredo JM, Muñoz Villa A, et al. 18q-syndrome and extraskeletal Ewing's sarcoma. J Med Gene. 1987;24(7):426-428.

16. Kaspers GJ, Kamphorst W, von de Graaf M, et al. Primary spinal epidural extraosseous Ewing sarcoma. Cancer. 1991;68(3):648-654.

17. Allam K, Sze G. MR of primary extraosseous Ewing sarcoma. Am J Neuroradiol. 1994;15(2):305-307.

18. Shin JH, Lee HK, Rhim SC, et al. Spinal epidural extraskeletal Ewing sarcoma: MR findings in two cases. Am $J$ Neuroradiol. 2001;22(4):795-798.

19. Mukhopadhyay P, Gairola M, Sharma M, et al. Primary spinal epidural extraosseous Ewing's sarcoma: report of five cases and literature review. Australas Radiol. 2001;45(3):372-379.

20. Gandhi D, Goyal M, Belanger E, et al. Primary epidural Ewing's sarcoma: case report and review of literature. Can Assoc Radiol J. 2003;54(2):109-113.

21. Harimaya K, Oda Y, Matsuda S, et al. Primitive neuroectodermal tumor and extraskeletal Ewing sarcoma arising primarily around the spinal column: report of four cases and a review of the literature. Spine. 2003;28(19):408-412.

22. Kogawa M, Asazuma T, Iso K, et al. Primary cervical spinal epidural Extra-osseous Ewing's sarcoma. ActaNeurochir (Wien). 2004;146(9):1051-1053.

23. Siami-Namini K, Shuey-Drake R, Wilson D, et al. A 15-year-old female with progressive myelopathy. Brain Pathology. 2005;15(3):265-267.

24. Athanassiadou F, Tragiannidis A, Kourti M, et al. Spinal epidural extraskeletal Ewing sarcoma in an adolescent boy: a case report. Pediatr Hematol Oncol. 2006;23(3):263-267.

25. Ozturk E, Mutlu H, Sonmez G, et al. Spinal epidural extraskeletal Ewing sarcoma. Journal of Neuroradiology. 2007;34(1):63-67.

26. Holland MT, Flouty OE, Close LN, et al. A Unique Case of Primary Ewing's Sarcoma of the Cervical Spine in a 53-year-old Male. A Case Report and Review of the Literature. 2015;2015:402313. 